\title{
The use of industrial wireless networks based on standard ISA- 100.11a and protocol WirelessHART in process control
}

\author{
A. V. Chupaev ${ }^{1}$, R. S. Zaripova ${ }^{2, *}, R . R$. Galyamov $^{1}$, and A. Y. Sharifullina ${ }^{1}$ \\ ${ }^{1}$ Kazan National Research Technological University, Kazan, Russia \\ ${ }^{2}$ Kazan State Power Engineering University, Kazan, Russia
}

\begin{abstract}
This article considers wireless technologies in the field of automation of technological processes and production in the energy, chemical, petrochemical and oil refining, gas, railway, mining, metallurgical and other industries where the use of wireless industrial devices is necessary due to specific features. Wireless technologies provide the ability to connect measuring devices directly to the wireless network with further transfer of information through a wireless gateway to an automated control system. This article will discuss the types of wireless networks that are classified according to the possible range of action (Wireless Wide area network, Wireless Local Area Network, Wireless Personal Area Network). The most common standards of organization of wireless industrial networks, including IEEE $802.11 \mathrm{~b} / \mathrm{g} / \mathrm{n}$, IEEE 802.15.1, IEEE 802.15.4, IEEE 802.16 e, will also be considered, including in the field of management of chemicaltechnological processes and productions. Data transmission protocols ISA-100.11.a and WirelessHART and a number of their features will be analyzed. The basic standard network structures based on the ISA100.11.a and WirelessHART protocols, their typical components, will be considered. The article provides an analytical comparison of the ISA100.11a and WirelessHART over the OSI network model layers, including the physical layer, the link layer, the network layer, the transport layer, the session layer, the presentation layer and the application layer (process control, management, security, application sublayer). The main stages of calculation of wireless industrial network are given, in particular: calculation of the network information load, calculation of the network's energy parameters, frequency-territorial planning, development of the communication scheme and interface with external networks. The formulas for calculating the network information load using Nazarov's methodology, calculating the network's energy parameters, taking into account the overall losses in the propagation of radio waves, including losses in free space, losses in partitions and walls inside the building, losses due to interference and signal fading are given.
\end{abstract}

\section{Introduction}

Currently, one of the main issues is the introduction of modern wireless technologies in the field of industrial automation. Creation of the reliable wireless process control systems is long overdue and must be accomplished not only at the production level, but the enterprise as a whole. Research in this area is carried out all over the world [1-3].

In the production of devices of automated control systems using wireless technology, takes into account the scope and conditions of their use. Taking these factors into account, it will be able use these devices in the following areas:

1) in chemical, oil and gas industry;

2) in the energy sector;

3 ) in the mining industry, etc.

Wireless technologies are able to integrate measuring devices into a wireless network with the possibility of further transmission of information into an automated control system [4-6].

\section{Methodology of research}

To set up a wireless network with the control system, it is necessary to take into consideration the specifics of network organization, analyse wireless data transfer technologies, calculate and design a wireless network to determine the locations of access points.

Wireless networks are classified by data transmission distance as follows:

1. WWAN (Wireless Wide Area Network) - cellular networks. The range is tens of kilometres (protocols GSM, CDMAone, iDEN, PDC, GPRS and UMTS).

2. WMAN (Wireless Metropolitan Area Networks) wireless networks of a city scale with a range of several kilometres (WiMAX protocol).

3. WLAN (Wireless Local Area Network) - a wireless local area network. The range is several hundred meters (protocols UWB, Wi-Fi).

4. WPAN (Wireless Personal Area Network) is a wireless network designed to organize wireless communication between various devices located on a

\footnotetext{
* Corresponding author: zarim@ rambler.ru
} 
limited area. Its radius of action can reach several tens of meters (RuBee, X10, Insteon, Bluetooth, Z-Wave, ANT, RFID, ZigBee, WirelessHART and ISA100.11a protocols).

The following standards were most widely used for organizing wireless communications, including at oil and gas chemical facilities:

1. IEEE $802.11 \mathrm{~b} / \mathrm{g} / \mathrm{n}$ is used for local wireless networks operating at distances from 100 to 300 meters, with data transfer rates of up to several megabytes per second.

2. IEEE 802.15.1 is used for Bluetooth communications, requiring very little energy and operating from 1 to 10 meters.

3. IEEE 802.15.4 is a low-speed communications standard designed to provide low power, short-range communications with low power consumption.

4. IEEE 802.16e is used for the WiMax communication protocol, which allows establishing communication at distances of 5-50 km, providing data transmission at speeds up to $72 \mathrm{MB}$ per second.

These standards use the $2.4 \mathrm{GHz}$ frequency band, which is used in the industry and is a special reserved ISM band (industrial, scientific, medical).

Versions of the IEEE 802.15.4 standard used in ISA100.11.a, WirelessHART, and are most commonly found in automatic control systems.

\section{Description}

WirelessHART is a network with a single-level coding (Flat Mesh Network), in which all devices are simultaneously sources and receivers of the signal [710]. During operation, the signal source (transmitter) sends an information to the nearest node, which transmits it to the next node. The transfer process continues until the information reaches the recipient.

The central component of such a network is the network administrator, who forms the network, establishes the communication structure and determines the backup paths, and also detects newly connected nodes to enter them into the existing network without interrupting its operation. It also implements the monitoring of all important functions of network nodes and carries out corrections in the event of a failure.

Coordination of packets in a network with singlelevel coding is performed using the Time Division Multiple Access (TDMA) method, which performs a very accurate synchronization of a radio station with a period of $10 \mathrm{~ms}$.

In order to reduce interference in the $2.4 \mathrm{GHz}$ band, the Frequency Hopping Spread Spectrum method (FHSS) is used.

WirelessHART devices use the same command structure as HART $4 \div 20 \mathrm{~mA}$. Therefore, WirelessHART applies only to devices that support the HART protocol.

The network ISA-100.11a includes: nodes with switching function (routers), nodes without switching function (input / output nodes), portable devices, backbone routers, system managers, security managers, and gateways.
Sensors and actuators are used as input/output nodes. Switched nodes can also be used as I/O devices.

In this network, data is transmitted from the source to the destination through several so-called "hops". At the same time, network routers are responsible for determining the correct destination.

The backbone router is also responsible for transferring data packets from one subnet through the backbone network to a destination in another subnet. The destination can be a gateway. It acts as an interface between the field network and the enterprise network.

The system administrator is the administrator of the entire network and is responsible for communication configuration (for example, resource allocation and scheduling), device management, and time control. The security manager is responsible for managing the security policy of the standard.

The essential difference between the ISA100.11a standard and the WirelessHART protocol is the possibility of using backbone data routing, as well as the use of individual field devices in switching mode, while WirelessHART implements the functions of transmitting and switching signals only at the field level [15]. The use of backbone routing improves the response time of autonomous field devices and reduces the load on their power supplies [11-14].

The time intervals in the ISA100.11a standard, unlike WirelessHART, are flexible and configurable. The ISA100.11a standard allows frequency hopping between channels, slowly changing frequencies from channel to channel and implementing a hybrid mode. Due to this, the ISA100.11a standard can work both with "active" devices that transmit data periodically and require time synchronization, as well as with "inactive" devices, in which the transmission of message packets occurs only when data changes or events that do not require synchronization of time come out.

To support uninterrupted and reliable operation of a wireless information network, it is necessary to calculate its characteristics, which consist of the following steps: calculation of the information load; calculation of the energy parameters of the network; frequency spatial planning; development of communication schemes and interfacing with external networks.

The calculation of the network information load can be made on the basis of the Nazarov method. With a known number of subscribers (sensors) at each object and the intensity of packet flows generated by subscribers of each service, the expected load on facility $\mathrm{i}$ of the k-th service can be determined by the expression:

$$
B_{i}^{k}=N_{i}^{k} \cdot T_{c}^{k} \cdot \gamma_{i}^{k} \cdot B_{\max }^{k}
$$

where $N_{i}^{k}$ is the number of subscribers to the $k$-th service at facility $i ; \gamma_{i}^{k}$ is the number of applications received from the subscriber of the $k$-th service per unit of time, $\gamma_{i}^{k}=1 / t ; T_{c}^{k}$ is the average duration of the 
communication session of the subscriber per unit of time; $B_{\max }^{k}$ is the maximum speed of the $k$-th service.

When calculating the energy parameters of the network, it is necessary to take into account the total losses in the propagation of radio waves. The size of losses $L$ is determined by summing losses in free space $L_{1}$, losses in partitions and walls inside the building $L_{2}$, losses due to interference and fading of the signal $L_{3}$ :

$$
L=L_{1}+L_{2}+L_{3}
$$

Also at this stage, it is necessary to calculate the communication range according to the method of calculating losses in free space (equations (3) and (4)):

$$
\begin{aligned}
& F S L=33+20(\lg F+\lg D) \\
& F S L=Y_{\partial B}-S O M
\end{aligned}
$$

where FSL is free space loss, $\mathrm{dB}$; $\mathrm{F}$ is the center frequency of the channel on which the communication system operates, MHz; D is distance between two points, $\mathrm{km}$.

The parameter SOM (System Operating Margin) is the stock, whisch in the power engineering of radio communications is usually taken equal to $10 \mathrm{~dB}$, since it is considered that it is sufficient for engineering calculation.

$Y_{\partial B}$ is a total system gain, which is determined by the equation:

$$
Y_{\partial B}=P_{t}+G_{t}+G_{\tau}-P_{\min }-L_{t}-L_{\tau}-L_{p}
$$

where $P_{t}$ is transmitter power, $\mathrm{dB} \mathrm{mW} ; G_{t}$ is the gain of the transmitting antenna, $\mathrm{dB} ; G_{\tau}$ is the gain of the receiving antenna, $\mathrm{dB} ; P_{\min }$ is receiver sensitivity at a given speed, $\mathrm{dB} \mathrm{mW} ; L_{t}$ is signal loss in the coaxial cable and connectors of the transmitting path, $\mathrm{dB} ; L_{\tau}$ is signal loss in the coaxial cable and connectors of the receiving path, $\mathrm{dB} ; L_{p}$ is the weakening of the electromagnetic wave in objects (loss).

By equating (3) and (4) and expressing D, it is possible to determine the communication distance of the projected network:

$$
D=10\left(\frac{F S L}{20}-\frac{33}{20}-\lg F\right)
$$

\section{Results}

Based on the results of calculations of the energy parameters of the network, as well as the characteristics of a particular object and the selected equipment, the procedure of frequency-territorial planning and the development of a scheme for organizing communications and interfacing with external networks have done.
Also, should be noted that in the process of network design, in order to eliminate mutual interference reduce the speed of information transfer, the neighboring points are tuned to non-overlapping frequencies of communication channels.

This setting is also carried out in order to increase the network coverage area, as well as to increase the speed of information transfer for each subscriber (sensor).

Conclusion:

Based on the presented algorithm, it is possible to calculate the main characteristics of a wireless industrial network, which will allow an increase of sampling points of technological parameters pulses due to the installation of measuring equipment (sensors) in difficult places.

\section{References}

[1] B.M. Vishnevsky, G.F. Gaykovich, Wireless sensor networks in industrial automation systems Electronics: Science, technology, business 1, 106-110 (2008)

[2] Y. Li, T. Txai, Wireless sensor networks and applications Springer (2008)

[3] M.S. Costa, J.L. Amaral, Analysis of wireless industrial automation standards: ISA-100.11a and WirelessHART (unpublished) Web Exclusive (2012)

[4] W. Gengyun, Comparison and Evaluation of Industrial Wireless Sensor Network Standards ISA100.11a and WirelessHART CHARMERS Master's thesis (2011)

[5] S. Petersen, S. Carlsen, WirelessHART Versus ISA100.11a IEEE Industrial Electronics (2011)

[6] D. Chen, M. Nixon, Wireless HART RealTime Network for Industrial Automation Springer (2010)

[7] S. Broadley, R. Karschnia, Using Wireless Transmitters in Single-Use Disposable Bioreactor Control IFPAC Conf ed M Nixon and T Blevins (2009)

[8] R.S. Zaripova, V.A. Belavin, The number of higher educational institutions. Problems of energetics 34, 93-98 (2006)

[9] L.R. Belyaeva, R.S. Zaripova, The number of higher educational institutions. Problems of energetics 12, 119-126 (2011)

[10] L.V. Plotnikova, I.I. Chilikova, Systematic approach to the assessment of energy complex efficiency for thermal energy production with heat power saving transformer turning on International Journal of Pharmacy and Technology 8(4), 26727-37 (2016)

[11] N.K. Petrova, H. Hanada, Computer simulating of stellar tracks for observations with the lunar polar telescope Planetary and Space Science 68, 86 - 93 (2012)

[12] N.K. Petrova, Y.A. Nefedyev The software complex for computer simulating the observation of stars from the lunar surface and calculating their selenographical coordinates SGEM 17(21), 687-694 (2017)

[13] N.K. Petrova, Y.A. Nefedyev Developing software processor to carry out analytical operations with trigonometric series by OOP method SGEM 18(21), 301-308 (2018) 
[14] L.V. Plotnikova, E.E. Kostyleva, The use of heat pump installations as part of waste energy convertion complexes in the joint generation of electrical and thermal energy IOP Conference Series: Earth and Environmental Science, International Scientific Conference "Efficient waste treatment 012067 (2018)

[15] L.V. Plotnikova, L.A. Kashipova, Modeling of heat and power system optimal structure using software Journal of Engineering and Applied Sciences 11(14), 3028-34 (2016) 